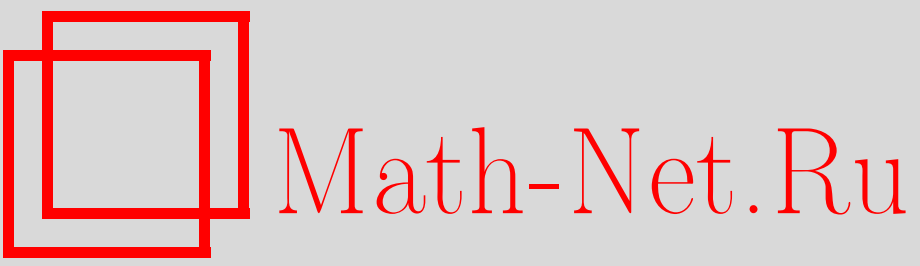

С. В. Людковский, Измеримость представлений бесконечномерных групп, $У М H$, 1996, том 51, выпуск 3, 205-206

DOI: https://doi.org/10.4213/rm986

Использование Общероссийского математического портала Math-Net.Ru подразумевает, что вы прочитали и согласны с пользовательским соглашением

http://www.mathnet.ru/rus/agreement

Параметры загрузки:

IP: 3.89 .185 .249

26 апреля 2023 г., 15:56:33 


\title{
ИЗМЕРИМОСТЬ ПРЕДСТАВЛЕНИЙ БЕСКОНЕЧНОМЕРНЫХ ГРУПП
}

\author{
С. В. Людковский
}

В данной статье доказано существование неизмеримых представлений для бесконечномерных групп Банаха-Ли (БЛ), ядерных групп Ли (ЯЛ) и групп диффеоморфизмов (ГД) $\operatorname{Diff}(l, b, M)$ локально компактных $C(l+1)$-многообразий $M$ (ЛКМ), где $0 \leqslant b<1, l \in \mathbb{N}$. Топология в $\operatorname{Diff}(l, b, M)$ индуцируется из соответствующего пространства Гёльдера $C(l, b, T M)$ (сечений расслоения $T M)$ с компактно-открытой топологией.

Лемма 1. Пусть $G$ - группа БЛ или ЯЛ над полем $K=\mathbb{R}, \mathbb{C}$ или конечныцм алгебраическим расширением $\mathbb{Q}_{p}$ с сепарабельной $C:=\bigcup\left\{U^{n}: n \in \mathbb{N}\right\}$, где $U=U^{-1} \ni е-$ окрестность нейтрального әлемента е. Тогда существует $\sigma$-аддитивная мера $m$ со значениями в $[0, \infty)$, определенная на борелевской $\sigma$-алгебре $B(G)$ и на ее пополнении $A(G, m)$ по мере $m$, причем $m:$ (1) радонова, (2) регулярна, (3) $m(S)=0$ для любого собственного топологического подмногообразия $S$ в $G\left(\operatorname{codim}_{K} S>0\right),(4) 0<m(C)<\infty$, (5) $m$ - действительная рассеянная (непрерьвная) мера.

Для доказательства отметим, что структура группы на $G$ согласована со структурой аналитического многообразия, пусть $X=T_{e} G$ - соответствующее сепарабельное банахово пространство (БП). На $X$ в случаях $\mathbb{R}, \mathbb{C}$ можно задать гауссову меру с помощью стандартной конструкции абстрактного винеровского пространства [3]. Аналогично в случае ЯЛ [4]. БП $X$ над $\mathbb{Q}_{p}$ изоморфно $c_{0}\left(\mathbb{N}, \mathbb{Q}_{p}\right)[5]$. На аддитивной группе $\mathbb{Q}_{p}$ имеется мера Хаара $w$ со значениями в $[0, \infty), w\left(\mathbb{Z}_{p}\right)=1, w(Y)>0$ для любого открытого $Y \neq \varnothing$ в $\mathbb{Q}_{p}$. Множества $S(j-1, n):=p^{j-1} \mathbb{Z}_{p} \backslash p^{j} \mathbb{Z}_{p}, S\left(n^{2}, n\right)=p^{n^{2}} \mathbb{Z}_{p} \quad \forall n \in \mathbb{N}, j \leqslant n^{2}$, являются открыто-замкнутыми в $\mathbb{Q}_{p}$. Можно задать следующее семейство мер

$$
v(n, E)=\sum\left\{a(j, n) w\left(p^{n^{2}-j}(E \cap S(j, n)) / w\left(p^{n^{2}-j} S(j, n)\right): j \in \mathbb{Z}, j \leqslant n^{2}\right\},\right.
$$

где $a(j, n)=\left(1-2^{-n}\right) 2^{n\left(j-n^{2}\right)}, E \in B\left(\mathbb{Q}_{p}\right)$. В $c_{0}$ существует ортонормированный (в $p$-адическом смысле) базис $\{f(j): j \in \mathbb{N}\}$ и ортогональные проекторы $\pi(n ; *)$ на $S^{\prime}(n)=$ $\operatorname{sp}\{f(1), \ldots, f(n)\}$. На $S^{\prime}(n)$ существуют меры $b(l, *)=v(1, *) \times \cdots \times v(l, *)$. Семейство мер $r\left(l, \pi_{n}^{-1}(E)\right):=b(l, E)$ согласовано и индуцирует $q$ на $X$. Подмножества вида $L:=\{x=$ $[x(j): j \in \mathbb{N}]: x(j) \in p^{k+k(j)} \mathbb{Z}_{p}$ при $j=1, \ldots, l$ и $x(j) \in p^{j} \mathbb{Z}_{p}$ при $\left.j>l\right\}$ компактны в $c_{0}$, причем $\forall \delta>0 \exists L: q\left(c_{0} \backslash L\right)<\delta$. По теореме Прохорова $q$ продолжается до $\sigma$-аддитивной меры на $A\left(c_{0}, q\right)$. Из сильной паракомпактности $G$ и существования гомеоморфизма открытых окрестностей $U$ в $G$ и $V \ni 0$ в $X$, получим $m$ на $G$, удовлетворяющую (1)-(5).

Далее, $\operatorname{Dif}(s ; l, b, M):=\left\{g \in \operatorname{Diff}(l, b, M) \mid \nabla^{j}(g(x)-x)=0 \forall 0 \leqslant j \leqslant s, x \in \partial M\right\}, s \leqslant l$, $\nabla^{0}:=1 ; \operatorname{Di}(l, b, M ; s):=\left\{g \in \operatorname{Diff}(l, b, M): \Delta^{j}(g(x)-x)=0 \forall x \in \partial M, j=0,1, \ldots, s\right\}$, $0 \leqslant 2 s \leqslant l$.

Лемма 2. Пусть $G=\operatorname{Diff}(l, b, M), \operatorname{Dif}(s ; l, b, M)$ uлu $\operatorname{Di}(l, b, M ; s) \partial л g C(\infty)-K M$ (или $\sigma$-компактного ЛКМ) $M$ или $G=G(t)$ из [1]. Тогда существует окрестность $W^{-1}=$ $W \ni е$, такая, что семейство әлементов $J:=\left\{g \in W:|r| \leqslant 1, r \in K\right.$ c $\left.h=g^{r} \in W\right\}$ плотно в $W$, где $\left\{g^{r}\right\}=:\langle g\rangle$ - локальные однопараметрические подгруппь.

Доказательство леммы проводится с помощью рассмотрения векторных полей на $M$ и соответствия между окрестностями 0 в $C(l, b, T M)$ и $e$ в $G$ (см. [8] и имеюшиеся там ссылки).

Tеорема 1. Пусть $M$ - компактное $C(k)$-многообразие, $\operatorname{dim} M=n, n \in \mathbb{N}, q \geqslant$ $[(n+1) / 2]+1+\operatorname{sign}(b), l \geqslant 2(3 q n+1), k \geqslant l+3 q+\operatorname{sign}(b), 0 \leqslant b<1, p^{\prime}-$ максимальное натуральное число такое, что $b\left(l, p^{\prime}\right) \leqslant[l / 2], b\left(l, p^{\prime}\right)=3 p^{\prime}-1$ nри четном $l$ и $b\left(l, p^{\prime}\right)=3 p^{\prime}$ при нечетном l. Тогда существует мера $m$, квазиинвариантная относительно $G(3):=$ $\operatorname{Diff}(l+3 q, b, M) \cap G(p, b, j)$ на (1) $\operatorname{Di}(p, b, M ; s)=: G(p, b, 1), 0 \leqslant 2 s \leqslant p ;(2) \operatorname{Diff}(p, b, M)=$ : $G(p, b, 2), p=0,1,2, \ldots, l ; j=1,2$. При этом в случаях (1), (2) $\exists$ окрестность $W \ni е$ 
в $G(p, b, j) c 0<m(W)<\infty$ такая, что $\forall \xi>0 u \forall \delta>0 \exists S \subset W$ - подмножество $c$ $m(W \backslash S)<\delta \cdot \max (1, m(W))$ и $\exists U-$ окрестность е, и $Y \subset U$ такие, что $m(U \backslash Y)<$ $\delta \cdot \max (1, m(U)), m(U)>0, U \subset W$ и $Y$ могут быть выбраны компактными, а $W, S$ и U симметричны (т.е. $\left.S^{-1}=S\right)$, причем каждая $\langle g\rangle:=\left\{g^{r}: r\right\}($ для $C(\infty)$-многообразия $M)$ пересекается с $S$ и $Y$ по интервалам, отличным от $\{e\}$ (т.е. $\{r \in(a, b)$ : $a<0<b\})$, функция плотности $t(g, x)=m_{g}(d x) / m(d x)$ равномерно непрерывна по $(g \in Y \cap G(3), x \in S) u|t(g, x)-1|<\xi \forall(g, x) \in(Y \cap G(3)) \times S$, причем $\forall g \neq e, g \in W \cap G(3)$ $\exists x \in W: t(g, x) \neq 1$. Здесь $m_{g}(Q):=m(g Q)$ для $Q \in A(G(p, b, j), m), j=1,2$.

Случаи 1 и 2 теоремы 1 для четных $l$ и $p=l$ содержатся в теоремах 1,2 в [6]. Для нечетных $l$ следует рассмотреть оператор $d \circ A$ вместо оператора $A$ из [6] и использовать теорему Пуанкаре, где $d$ - внешнее дифференцирование. При $p<l$ можно рассмотреть счетное всюду плотное подмножество точек $\{z(i): i \in \mathbb{N}\}$ в $M$ и соответствующие отображения вычисления $F(i, g):=g(z(i))$, тогда можно задать подмножества $W(k, p, c ; f):=\{g \in W(p)$ : $\left.|F(i, g)-F(i, f)|_{C(p, b, T M)} \leqslant c, i=1, \ldots, k\right\}$, где $c>0, k \in \mathbb{N}, f \in W(l), W(l)=W(p) \cap$ $G(p, b, j), W(l)$ - окрестность $i d$ в $G(l, b, j), m(l, *)$ - мера на $W(l)$. Тогда можно определить $m(p, W(k, p, c ; f)):=m(l, W(k, l, c ; f))$. Поскольку семейство $\{W(k, p, c ; f): k \in \mathbb{N}, c>0$, $f \in W(l)\}$ порождает $B(W(p))$ с помощью счетного числа объединений и дополнений, то $m(l, *)$ индуцирует $m(*)=m(p, *)$ на $G(p, b, j)$, так как топология на $G(l, b, j)$ сильнее, чем на $G(p, b, j)$.

Теорема 2. Пусть $G$ - нетривиальная группа БЛ или ЯЛ с $m$ из леммы 1, или $G(p, b, j)$ с $m$ из теоремь 1 , или неархимедова $G(t)$ из [1], или недискретнал ЛК группа с левой мерой Хаара $m$. Тогда $G$ имеет топологически неприводимые унитарнье представления $T: G \rightarrow U(H)$, не являющиеся слабо измеримыми. В случаях коммутативной $G$ или ГД она имеет неизмеримые характеры. Кардинальное число семейства таких представлений равно $2^{\text {card } G}$.

Доказательство проводится аналогично [2] с помощью построения алгебраических автоморфизмов $f: G \rightarrow G, f(G)=G$, таких, что для некоторой симметричной окрестности $U \ni$ e с $0<m(U)<\infty, U \subset C$ для любых открытых $E$ и $F$ в $U$ выполняется: (1) $\operatorname{card}(f(E) \cap F)=\mathfrak{C}$. В случае групп Ли в шаре $V$ с центром в $0, V \subset X$, рассматривается топология ежа и соответствующая топология в $U$ [7]. Причем в (1) накладывается дополнительное условие, что $E$ и $F-$ открытые подмножества в $J$, где $J$ - объединение счетного семейства локальных однопараметрических подгрупп из $U$, причем $J$ плотно в $U$. Для $G(p, b, j)$ и $M$, не принадлежащих классу $C(\infty)$, или $G(t)$ из [1], элементы бесконечного порядка плотны в $U$ и мошность их семейства равна $\mathfrak{C}$, так как существует подходящая последовательность $C(\infty)$ многообразий $\{M(i): i \in \mathbb{N}\}$, сходящаяся равномерно к $M$ в $\mathbb{R}^{2 n+1}$, что дает $f$. Если представление $T: G \rightarrow U(H)$ - измеримое и нетривиальное, то $T \circ f$ - неизмеримое слабо.

\section{СПИСОК ЛИТЕРАТУРЫ}

[1] Людковский С. В. // УМН. 1996. Т. 51. № 2. С. 169-170. [2] Людковский С. В. // Матем. сб. 1995. Т. 186. № 2. С. 83-92. [3] Го Х. Гауссовы меры в банаховых пространствах. М.: Мир, 1979. [4] Гельфанд И. М., Виленкин Н. Я. Обобщенные функции. Т. 4. М.: Физматлит, 1961. [5] van Rooij F. C. M. Non-Archimedean functional analysis. New York: Marcel Dekker, 1978. [6] Шавгулидзе Е. Т. // ДАН СССР. 1988. Т. 303. №4. С. 811-814. [7] Энгелькинг Р. Общая топология. М.: Мир, 1986. [8] Lang S. Differential manifolds. Berlin: Springer-Verlag, 1985. 